\title{
Divisional Sessions
}

During three afternoons of the EPS General Conference, participants attended parallel sessions which had been organized by the Specialized Divisions of the Society. In this section, we carry reports which have been contributed mainly by the Chairmen of sessions.

\section{Atomic and Molecular Physics}

(report contributed by $H$. van Regemorter, Chairman of the EPS Specialized Division on this topic)

Two afternoon sessions were devoted to lectures on atomic and molecular physics. In the first lecture, M.J. Seaton (London) considered the problems of Bound States and Continuum States of Atomic Systems together, since there was much to be gained by this approach. He considered techniques for obtaining analytic continuation of the scattering matrix, so as to underline the connection between atomic structure calculations and problems of electron-atom scattering. B. Fastrup (Aarhus) spoke about Violent Ion-Atom Collisions at Moderate Nuclear Velocities which have proved to be very efficient for the ionization of inner atomic shells and for producing $\mathrm{x}$-rays. In his talk Spectroscopy with Lasers, W. Demtröder (Kaiserslautern) reviewed the performances of wideband tunable dye lasers. $\mathrm{He}$ explained the basic principles of saturation spectroscopy, level crossing and double resonance spectroscopy with lasers and photon echotechniques. Demtröder also described the investigation of ultra-fast transient phenomena by pico-second pulse lasers and the contribution of laser spectroscopy to explaining collision processes and relaxation phenomena.

Opening the second afternoon session, G. zu Putlitz (Heidelberg) spoke about Optical Pumping of lons. He underlined the drastic differences of the sizes of effects observed using ions from the equivalent processes with neutral atoms.

Relativistic Effects in Atomic Spectroscopy were considered by S. Feneuille (Orsay). Amongst the topics examined by new formalisms and computational techniques were transition probabilities, hyperfine structures, and isotope mass shifts. The only paper on molecular physics was given by K. Dressler (Zürich) who discussed the information that may be obtained from the study of Optical Spectra of Trapped Atoms and Molecules in low temperature molecular crystals.

\section{Condensed Matter and Low Temperature Physics}

(report on the first session contributed by $N$. Kurti and on the second session by A. Guinier, Chairmen)

\section{First session}

In the first lecture, T. Springer (Jülich) reviewed recent developments in high resolution neutron spectroscopy. A backscattering spectrometer has been developed so that misalignment hardly affects the resoIution. With this apparatus used at a high flux beam reactor, an energy resolution of $5 \times 10^{-7} \mathrm{eV}$, corresponding to a frequency resolution of about $10^{8} \mathrm{~s}^{-1}$, has been achieved - a more than hundredfold improvement on previous instruments. However the method has the drawback that, since the Bragg angle is fixed, the energy can be varied only slightly by Doppler motion. The analysis of the spectra permits the determination of rotational or translational relaxation times in the region of $10^{-13}-10^{-8} \mathrm{~s}$, thus providing an overlap with nuclear magnetic resonance and ultrasonic methods. Results of two series of investigations were given :

(a) Random rotations in molecular crystals e.g. in (solid) methane.

(b) The diffusion of hydrogen in the $\mathrm{Nb}-\mathrm{H}$ system. Measurements were done both above the 'critical' point, where dissolved hydrogen behaves like a gas, and in the 'two-phase' region. Agreement with the measurements of the macroscopic diffusion coefficient was good above the critical point, while an apparent disagreement at the critical point could be resolved by allowing for the fact that, unlike the jump-times obtained by neutron spectroscopy, the macroscopic diffusion constants are affected by the variation of the chemical potential with concentration.

In the second lecture, J. Labbé (Orsay) gave an account of theoretical investigations to explain the high critical temperatures for superconduction of A-15 compounds (e.g. $\mathrm{Nb}_{3} \mathrm{Sn}$ or $\mathrm{V}_{3} \mathrm{Si}$ ), their anomalous mechanical be- haviour and the connection between these two. It has been shown that there is a gradual softening of the shear modulus as the temperature is lowered and that, at a temperature slightly above the critical point, the cubic modification becomes unstable and there is a transition into a tetragonal phase. An essential feature of the theory is that in these compounds the transition metal ions are arranged in non-interacting linear chains, and this results in a high degeneracy of the electronic states. The degeneracy is partially lifted by the shear strain : a Jahn-Teller effect affecting a band rather than localized ions. There is a sharp singularity in the electronic density of states close to the Fermi level, and it is this, rather than a strong phonon-electron coupling, that is proposed to be responsible for the high critical temperatures of these compounds.

D. Shoenberg (Cambridge) gave a comprehensive account of the past, of the present, and of the future prospects of the de Haas-van Alphen effect, i.e. the oscillatory field dependence of magnetization in metals. Thanks to the theoretical interpretation by Onsager and by I.M. Lifshitz, the effect has become perhaps the most effective and most widely used method for the study of the Fermi surfaces of metals. The ready availability of very low temperatures, of intense and stable (or slowly and regularly changing) magnetic fields produced with superconductors greatly helped to extend the scope and the applicability of the method.

While Fermi surface determinations rely on the study of the frequencies of de Haas-van Alphen oscillations, the measurements of their amplitudes permit collection of information about the relaxation times and the spin properties of electrons. In particular, measurements have been done on the effect of magnetic impurities in metals (Kondo-effect) and, most recently at Cambridge, on electronic g-factors.

D. Shoenberg's lecture was a masterpiece of lucid and lively exposition and some of his felicitous turns of phrase have further enriched the vocabulary of Fermiologists. 'Swimming in the Fermi sea' is a well-known expression, but Shoenberg, in his opening remarks extended these sporting activities by saying that he would only 'skate on the Fermi surface' and later, to characterize the ever-increasing refinements of experimental techniques, he left the world of sport for that of cosmetics and said that one can now 'see every wrinkle on the face of the Fermi surface'. 


\section{Second session}

A problem still far from being solved satisfactorily is the atomic structure of solids. J.L. Beeby (Harwell) showed that, in order to use the considerable amount of experimental data now available (from LEED, scattering of atoms), decisive progress with theoretical techniques is needed.

Superplasticity is one of the fields of metal physics in full activity at the moment. D.J. Dingley (Bristol) summarized the well-established facts, and presented the general features of a new theory of Ashby which seems to be very promising. Finally, G. Amsel (Paris) demonstrated the possibilities of his method of surface analysis by observation of nuclear interactions. Although the method can only be used in specially equipped laboratories, it has unique features (particularly, very high sensitivity) which make it very important for solving a wide variety of problems of physical chemistry, surface technology, metallurgy, etc.

\section{Computational Physics}

(a general report by M.R. Feix (Chairman of first session), and a report on second session by H. Thomas (Chairman of second session))

\section{General report}

The two afternoons organized by the Computational Physics Group were joint sessions respectively with the Advisory Committee on Applied Physics and Physics in Industry and with the Physics in Astronomy Division. Moreover, no fewer than three papers were devoted to plasma physics.

The many aspects of the 'computational physics' field were apparent. In the conceptually simple problem tackled by E.T. Ferguson (Eindhoven) (electron gun design and electron microscopy), the computer is an excellent working tool to predict and improve the performances of the design apparatus. Optimization of spaceflights, dealt with by $\mathbf{H}$. Göllnitz (Munich), also has interesting problems well simulated on the computer. If we add accelerator studies, as described by N. Vogt-Nilsen (Geneva), we find that the 'orbits' problem (including configuration of magnetic fields in magnet design for fusion and accelerator machines) is well suited to the computational approach used in practical industrial problems. The optimistic view that the computer might now be used for materials research was given by J. Treusch (Dortmund). Nevertheless, in solid state studies (band structure calculations by Treusch and phonon spectra by K. Knorr (Grenoble)) the theory is still very crude (using, for example, the one electron approximation), and experimental results have to be included in the construction of working models. On the other hand, the computer inspires theoreticians to understand some highly sophisticated and simplified Ising models, as described by $\mathbf{T}$. Schneider (Zürich). Finally, in the 'phase space problems' (galactic, plasma, and statistical mechanics) described by M.R. Feix (Orleans) and D. Biskamp (Garching), and also in hydrodynamics (R. Sadourny (Paris)), many models have been worked out. The computer brings completely new possibilities. For example, we can follow the behaviour of one 'test particle', or we can 'experiment' with different initial conditions for the development of spiral arm structures in galaxies.

Of course, numerical difficulties do exist (especially in the phase space problems), and we must look for the best strategy to reduce, from $10^{24}$ to $10^{5}$, the number of degrees of freedom in order to adjust it to the storage capacity of the computer. This induced one speaker to point out a 'pseudophysics', and that we must be careful to keep this 'pseudo-physics' close enough to the real world.

A word on the display problem - we obtain a lot of information, and we must use as much as we can. Films actually seem the best way to grasp the global situation quickly.

The last plenary talk (by $\mathbf{Y}$. Le Corre) was on the computer and the teaching of physics with some classical uses of the computer (a film of a Schrödinger solution). The speaker outlined the more ambitious task of arranging that the computer should 'talk physics' with students - a field where mathematics, logic, physics and psychology combine and fully exhibit the various uses of the computer.

\section{Second session}

The first three lectures of this session gave reports on the use of computational methods in various areas of solid state physics. J. Treusch (Dortmund) gave a concise survey of band structure calculations, and discussed the advantages and failures of the various methods with respect to computer application. A summary of results representative of the present state of the theory demonstrated how these calculations may be used for a better understanding of properties of ordered and amorphous semiconductors, and for a pictorial representation of metallic binding by contour plots of the charge density. Treusch expressed
New

North-Holland publications

\section{Foundations of Electrodynamics}

by S.R. de GROOT and L.G. SUTTORP, Institute of Theoretical Physics, University of Amsterdam.

1972. 535 pages. Dfl. 140.00 (ca. $\$ 45.00$ ) ISBN 0720402484

Contents: Part A: Non-Relativistic Classical Electrodynamics. Particles: their fields and motion. Statistical description of fields and matter. Part B: Covariant Formulation of Classical Electrodynamics. Charged point particles. Composite particles. Covariant statistics: The laws for material media. Part C: Non-Relativistic Quantum-Mechanical Electrodynamics. The Weyl formulation of the microscopic laws. Quantum-statistical description of material media. Part D: Covariant and Semi-Relativistic Quantum-Mechanical Electrodynamics. Dirac and Klein-Gordon particles in external fields. Semi-relativistic description of particles with spin. Semi-relativistic quantum statics of spin media. Index.

\section{Microwave Ultrasonics in Solid State Physics}

by J.W. TUCKER, University of Sheffield, U.K., and V.W. RAMPTON, University of Nottingham, U.K.

1972. 428 pages. Dfl. 130.00 (ca. $\$ 42.00$ ) ISBN 0720402549

Contents : Introduction. Elasticity and lattice dynamics. The generation and detection of microwave ultrasonics. Attenuation of ultrasonic waves in dielectrics. Ultrasonic waves in ferromagnetic materials. Acoustic paramagnetic resonance. Ultrasonic waves in semiconductors. Microwave ultrasonic propagation in metals. The interaction of light with microwave ultrasonic waves. Appendices. Author index. Subject index.

\section{Progress in Optics, Volume $X$}

edited by E. WOLF, Department of Physics and Astronomy, University of Rochester, New York. 1972. 400 pages. Dfl. 100.00 (ca. $\$ 31.25$ ) ISBN 0720415101

Contents : Bandwidth compression of optica images (T.S. Huang). The use of image tubes as shutters (R.W. Smith). Tools of theoretical quantum optics (M.O. Scully and K.G. Whitney). Field correctors for astronomical telescopes (C.G. Wynne). Optical obsorption strength of defects in insulators (D.L. Dexter and D.Y. Smith). Elastooptic light modulation and deflection (E.K. Sittig). Quantum detection theory (C.W. Hellstrom) Acknowledgement. References. Author index. Subject index.

Sole distributors for the U.S.A. and Canada : American Elsevier Publlshing Company, Inc.,
52 Vanderbilt Avenue, New York, N.Y.' 10017. 
his view that calculations of this kind may in future become a valuable tool in materials research. K. Knorr (Grenoble) reported on a method to compute phonon spectra of superconductors from measurements of their tunneling characteristics. This method requires the solution of a system of coupled integral equations by a complex iteration scheme. The outline of a computer programme was given : it calculates the electronic density of states by iterating an originally guessed phonon spectrum until experimental and computed data match. Knorr illustrated that this method is so sensitive that it shows the shift of the phonon spectrum to lower frequencies for disordered samples. T. Schneider (Zürich) presented a film on a computer simulation of the dynamics of a first order phase transition in an Ising model just below $T_{c}$. The time evolution of the microscopic state of the system from a given initial state and, in particular, the gradual reversal of magnetization under sudden reversal of the external field could be traced on the computer display. The opinion was expressed that such computer investigations of an idealized system might inspire further studies of the dynamics of clusters in the process of phase nucleation.

\section{Nuclear Physics \\ (report contributed by W. Greiner, Chairman)}

In the session on Nuclear Physics, four speakers covered a large field ranging from nuclear structure of neutron rich isotopes via theoretical predictions on superheavy nuclei and large-scale shell model calculations to three-body reactions. R. Klapisch (Orsay) reported on exotic nuclei off the stability line, which are produced by high energy ( $24 \mathrm{GeV}$ ) proton bombardment of heavy nuclei (e.g. uranium). The resulting spallation products are analyzed with the ISOLDE mass spectrometer at CERN and a number of neutron rich, light isotopes are found. One of the exciting examples is the discovery of ${ }^{32} \mathrm{Na}$ and ${ }^{33} \mathrm{Na}$ (the most stable $\mathrm{Na}$-isotope is ${ }^{23} \mathrm{Na}$ !). This allows a check of semiempirical mass formulae and particular terms (symmetry energy) of the exotic nuclei.

W. Szymanski (Warsaw) summarized the various attempts at the prediction of superheavy nuclei. The theoretical concepts (shell corrections, collective potentials and collective masses) were presented in a fine talk, together with the most recent expectations on the lifetimes of the nuclei of the $(Z=114)$ island. During the talk, but also in the following lively discussion, the critical aspects of the calculations came up, in particular, the zero-point energy problem and the effect of the $\gamma$-degree of freedom (oblate fission). Consideration of these latter effects leads to a more realistic estimate of the lifetimes of superheavy elements of the order of $1-10^{4}$ years.

A great demonstration of 'computer physics' was given through the presentation of a method for large-scale shell model calculations by I.M. Irvine (Manchester). The method of constructing an optimal - though truncated - Hilbert space for shell model calculations by repeated application of the nuclear shell model Hamiltonian on a model state, followed by orthogonalization of the set, is appealing. But it needs to be seen how the practical results will turn out.

It was unfortunate that I.C. Organesjan (Dubna) could not come to deliver his talk on 'fission induced by heavy ions'. His replacement by W. Sandhas (Mainz) allowed for a very clear presentation of the latest theoretical and experimental results on the threebody-problem. The main physical insight resulting from several works on this subject is the equality of the $p-n$ and $n-n$ scattering length and thus of the charge independence of nuclear forces.

\section{Plasma Physics}

(report on the first session contributed by $B$. Lehnert, and on the second session by C.M. Braams, Chairmen)

\section{First session}

Of the two sessions organized by the Plasma Physics Division, the first concentrated mainly on the subject of cosmic plasmas. The session started with a lecture by R. Lüst (Garching) on Plasma Experiments in Space. Until quite recently, theory in combination with astronomical observations and laboratory model experiments had comprised the only methods of tackling the problems of cosmic plasma physics. During the last decade, however, space techniques have opened up the new possibility of direct study of the cosmical plasmas. By such experiments, it is now possible to measure the physical parameters of extra-terrestrial plasmas, as well as to investigate their general properties in a way which is impossible in the laboratory according to scaling laws. In the lecture, several examples were given of plasma experiments in space, such as the artificial clouds released by the MaxPlanck-Institut for Extraterrestrial Physics. These clouds were launched in order to study their interaction with the surrounding plasma in space and with arising electric and magnetic fields. They comprised barium and were ejected mainly at a height of $200 \mathrm{~km}$, but also at $1000,2000,33000$ and $74000 \mathrm{~km}$. Simultaneous measurements of electric field were performed at these altitudes. From the measurements, the corresponding plasma 'winds' were calculated, and the electromagnetic dynamo action and some of the instability modes of extraterrestrial plasma could be studied.

When research on controlled fusion started in the early 1950s, it was based mainly on the relatively simple theoretical models developed at that time in plasma physics and magneto-hydrodynamics in connection with applications in cosmical physics. The confrontation between these models and laboratory experiments on magnetically confined, fully ionized plasmas revealed large discrepancies, thus initiating many years of extensive work on the refinement and extension of plasma theory. As described by $\mathbf{B}$. Coppi (Cambridge, USA), recent results of laboratory experiments and parallel theoretical investigations have led to the understanding of processes such as instability phenomena and transport processes resulting from plasma turbulence. These processes are directly relevant not only to fusion research but also to the interpretation of astrophysical phenomena, such as those involving the emission of energetic particles and radiation. Examples of models were given and of investigations of solar flares, x-ray stars and pulsars.

The problems of wave propagation in plasmas have now been treated extensively for 30 years and are predictable in linear approximation, from both the theoretical and the experimental points of view. The nonlinear processes affecting wave propagation are less well-known.

Recent progress in this vast field, which for the moment attracts considerable interest among scientists, was outlined by J.L. Delcroix (Orsay). The lecture treated the problems of wave-wave coupling and decay with associated instabilities, particle-wave coupling and particle trapping by large-amplitude waves, as well as the formation of solitary waves (solitons) and shock waves.

\section{Second session}

The second session on plasma physics contained two talks : Collisionless Shocks and Plasma Turbulence by J.W.M. Paul and Numerical Simulation of Plasma Phenomena by K.V. Roberts, both from Culham Laboratory. These gave the audience an excellent 
introduction to important trends in plasma physics: the study of turbulent transport phenomena and the introduction of computational physics in support of plasma theory and experiments.

Paul led up to his subject by raising the question : 'How can shocks dissipate without particle collisions?' In high temperature plasmas, interaction between charged particles very often proceeds through non-linear interactions of waves, that is, through collective phenomena, rather than through binary collisions. The theory for weak turbulence provides estimates of effective transport coefficients in that regime; no such theory is available for the strong turbulence regime. Scattering of laser light and emission of electromagnetic radiation from turbulent plasmas show fluctuation spectra in general agreement with Kadomtzev's theory, which is also supported by measurements of plasma resistivity. Thus, laboratory plasma physics has laid a basis for understanding what has been named 'anomalous' but appears to be rather normal behaviour, also of cosmical plasmas.

Roberts mentioned a number of areas in which computational physics is supporting plasma theory and experiment, and identified three fields, namely initial-value problems, equilibrium configurations, stability of equilibrium states, in which major computational efforts have taken place. Further, in direct support of the design of experiments, magnetic fields, particle orbits and optimized machine parameters have been computed by several groups.

One- and two-dimensional plasmas can be simulated by direct integration of equations of motion for a large number of particles. In three dimensions, the computer time required would be prohibitive, so that one has to resort to a macroscopic description of the plasma. The speaker indicated conditions under which results become inaccurate or numerical instabilities occur, and went on to suggest various methods to eliminate degrees of freedom or to modify space- or time-scales in order to render problems amenable for computer simulation. Robert's talk was followed by a film showing the results of computations on some hydrodynamic vorticity phenomena which are analogous to the motion of magnetized plasmas in phase space. It was striking to see instabilities leading to the formation of new and apparently stable equilibria, a phenomenon also seen in certain fusion experiments.

\section{Quantum Electronics}

(report of first session by W. Kaiser (Chairman) and of the second session by K.P. Meyer (Chairman) and $M$. Keller)

\section{First session}

High Power Double Discharge TEA Lasers. M. Michon (Marcoussis) presented detailed theoretical and experimental investigations of high power $\mathrm{CO}_{2}$ lasers. The TEA laser system of a marketed device produced $300 \mathrm{MW}$ for $60 \mathrm{~ns}$. Monomode operation was achieved in a 12-element system where the successive elements are twisted by $30^{\circ}$ with respect to each other. The speaker predicted a next generation device to emit $10^{4} \mathrm{~J}$.

Tunable Infrared Sources. S.D. Smith (Edinburgh) discussed the various merits of four tunable infrared light sources known at present: the laser diodes, parametric oscillators, stimulated light scattering by polaritons and by spin reversal. Special emphasis was given to the last subject, the speaker's own field of research. Examples of the high resolution of the new tunable infrared light sources were presented and a revolution in infrared spectroscopy was predicted.

Dye Lasers. F.P. Schäfer (Göttingen) gave a detailed account of the various dye families which are useful for laser application. Continuous wave (CW) dye lasers have been pumped with other lasers. Pumping with incoherent light sources is actively pursued at present for CW operation in the not too distant future. The applications of dye lasers in integrated optics, in holography and saturation spectroscopy were discussed.

Picosecond Phenomena and their Measurement. D.J. Bradley (Belfast). Recently laser dyes have produced mode-locked pulse trains with light pulses in the picosecond range. The two photon fluorescence techniques represent a cheap and quick method to estimate the pulse duration. But care has to be taken in the interpretation of simple pictures. A more direct investigation of the very short light pulses is made possible by an electrooptical streak camera. The performance of this camera was discussed.

\section{Second session}

In the first lecture, Semiconductor Lasers, M.H. Pilkuhn (Stuttgart) emphasized the point that through the advent of the double heterojunction structure, continuous operation at room temperature of these devices became possible. This breakthrough, together with the progress made in related fields like detectors, modulators and fibre optical transmission lines, makes the semiconductor laser very attractive and very promising for communications systems. The remaining drawback is the relatively short lifetime of the laser due either to catastrophic self-damage or to slow degradation effects.

The paper Self-focusing and Selftrapping given by 0 . Svelto (Milan) dealt with a group of nonlinear optical effects. If the polarization induced in a material is described by a series expansion in terms of powers of the field of the incident light wave, the terms containing the third and higher odd orders of the field can be shown to induce polarization source terms at the fundamental frequency, thus giving rise to an action of the light wave upon itself. These self-action effects are: self-focusing, self-steepening, self-phasemodulation and self-rotation of polarization. On the other hand an intense light field induces nonlinearities in the material: changes in polarizability and density. Since all these effects are not yet completely understood there is plenty to be done in future work of both theoretical and practical importance.

The last paper Laser Produced Plasmas and Fusion by S.A. Ramsden (Hull) showed that the decision to continue research in spite of the deceiving results obtained in 1964 was rewarded. The first evidence of laser induced nuclear fusion dates back to 1968. Since then results steadily improved and further enhancement can be expected (eg by using $\mathrm{CO}_{2}$ lasers). The most important point is that theoretical values of the laser power needed for a breakeven controlled thermonuclear reaction show a tendency to decrease whereas the laser power has achieved increases from year to year.

Reports on sessions on Physics in Astronomy and High Energy and Particle Physics will be published when available.

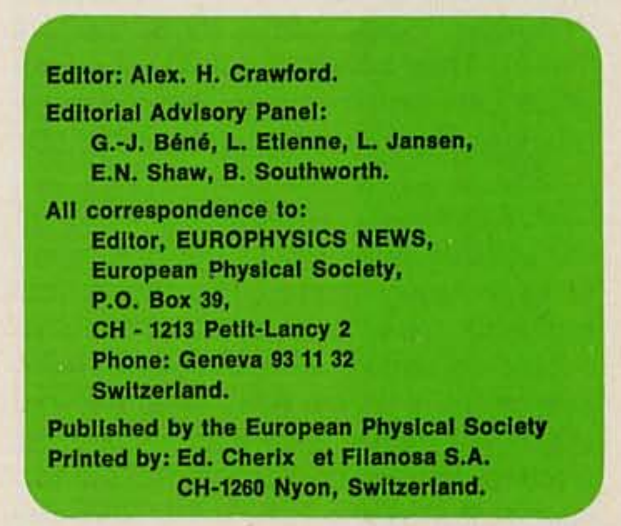

